\title{
Comparando os Impactos da Percepção do Chamado Ocupacional na Vida Pessoal, Profissional e na Saúde dos Funcionários Públicos
}

\author{
http://dx.doi.org/10.21527/2237-6453.2020.51.281-296 \\ Recebido em: 21/3/2019 \\ Aceito em: 5/12/2019 \\ Bruno Felix, ${ }^{1}$ Luiz Felipe Magnago Blulm²
}

\begin{abstract}
RESUMO
O objetivo deste trabalho é avaliar como diferentes percepções acerca do chamado ocupacional podem impactar a vida pessoal e profissional e a saúde psicológica dos funcionários públicos no contexto socioeconômico brasileiro. A amostra foi dividida em três grupos: os que (1) percebem o chamado ocupacional e atendem a ele, os que (2) o percebem, mas não conseguem atender, e os que (3) não possuem nenhum chamado ocupacional. O questionário foi aplicado numa amostra de 274 participantes. Os dados foram rodados com Anova e depois foi realizado um teste Duncan para evidenciar quais grupos se diferenciavam. Parte dos resultados foram consistentes com a literatura existente sobre o chamado ocupacional e com a teoria da autodeterminação. Diferentemente dos estudos realizados em outros contextos, entretanto, os resultados apontaram não existir diferenças significativas entre os grupos que possuem um chamado não atendido e os que não possuem nenhum chamado. A contribuição deste artigo está em realizar a comparação dos efeitos citados em um país com contexto socioeconômico diferente dos explorados na literatura até o momento.
\end{abstract}

Palavras-chave: Chamado ocupacional. Funcionários públicos. Brasil.

\section{COMPARING THE IMPACTS OF THE PERCEPTION OF THE OCCUPATIONAL CALL INTO PUBLIC SERVER'S HEALTH, PERSONAL AND PROFESSIONAL LIVES}

\section{ABSTRACT}

The aim of this paper is to evaluate how different perceptions about that occupational call can impact one's personal and professional lives and also its psychological health, specifically on the Brazilian socioeconomic context. The sample was divided into three groups: the ones who have an occupational calling and respond to it, the ones who have an unanswered calling and others who doesn't have an occupational calling. A survey was answered by a sample of 274 participants. Data was analyzed on ANOVA and then a Duncan test was made to put in evidence which groups difference themselves. Part of the results were considered consistent according to the previous Calling literature and the Self-determination theory. However, differently from studies taken place in other contexts, our results point that there were no meaningful differences between the groups which have an unanswered calling and the ones which have no calling at all. The main contribution of this article it to explore a singular context among the existent literature.

Keywords: Occupational call. Public servers. Brazilian.

\footnotetext{
${ }^{1}$ Doutor em Administração pela Universidade Presbiteriana Mackenzie. Professor da Fundação Instituto Capixaba de Pesquisa em Contabilidade, Economia e Finanças (Fucape).bfelix@fucape.br

${ }^{2}$ Mestre em Administração de Empresas pela Fundação Instituto Capixaba de Pesquisa em Contabilidade, Economia e Finanças (Fucape). Professor das Faculdades Integradas de Aracruz (Faacz). luizfelipemb17@gmail.com
} 
Nas últimas décadas o ambiente corporativo em culturas ocidentais tem promovido uma narrativa a qual incentiva que trabalhadores busquem em suas carreiras desenvolver uma atividade dotada de significados e realizações (STEGER; DIK; DUFFY, 2012). Tal narrativa tem estimulado uma crescente ressignificação do papel do trabalho e levado pessoas a esperarem de suas carreiras resultados que transcendem as relações de troca entre trabalho e recompensas financeiras estabelecidas em abordagens econômicas (ARNOLD et al., 2007; ROUSSEAU; HO, GREENBERG, 2006). Assim, pode-se notar que alguns indivíduos têm buscado mais que uma ocupação profissional; cresce, então, a busca por um chamado ocupacional (BERG; GRANT; JOHNSON, 2010; WRZESNIEWSKI, 2003). Neste estudo adota-se a definição de chamado ocupacional como uma ocupação em que uma pessoa se sente motivada a buscar, de forma a construir uma carreira que seja prazerosa, dotada de significado e uma parte central de sua identidade (WRZESNIEWSKI et al., 1997).

Estudos anteriores têm mostrado efeitos positivos de se possuir um chamado profissional atendido. Por exemplo, pesquisas indicam que indivíduos que relatam viver um chamado ocupacional possuem um maior bem-estar (DUFFY; DIK, 2013; DUFFY et al., 2017) e uma maior preparação na carreira (HIRSCHI; HERRMANN, 2013; PRASKOVA; HOOD; CREED, 2014). A literatura, entretanto, também mostra que existem aspectos prejudiciais em se ter um chamado ocupacional e não conseguir atender a ele. Estudos recentes evidenciaram que possuir um chamado ocupacional não atendido tende a ser mais prejudicial do que não perceber nenhum chamado, e que ambas as situações não trazem os benefícios que ter um chamado ocupacional atendido pode proporcionar (GAZICA; SPECTOR, 2015). A maior parte dos estudos sobre o tema foram realizados em países desenvolvidos (DOUGLASS; DUFFY; AUTIN, 2016; DOMENE, 2012; HIRSCHI; HERRMANN, 2013). Até então os estudos feitos nos países emergentes foram realizados na China e na Índia; países orientais que apontaram resultados diferentes dos realizados nos países desenvolvidos, trazendo como uma possível explicação as diferenças socioeconômicas entre eles (ZHANG et al., 2015; DOUGLASS; DUFFY; AUTIN, 2016). Pesquisas anteriores também mostraram que um chamado pode ser experimentado de forma distinta em campos diferentes, como contextos socioeconômicos, gêneros, níveis de educação e etnias (AUTIN et al., 2017).

Desta forma, a lacuna que este estudo propõe preencher é comparar indivíduos com diferentes percepções acerca do chamado ocupacional em um país com o contexto socioeconômico diferente, ou seja, um país ocidental emergente. Assim, o objetivo deste estudo é avaliar se indivíduos que possuem um chamado ocupacional atendido, um chamado ocupacional não atendido e aqueles que consideram que não possuem um chamado ocupacional apresentam diferenças significativas em termos de variáveis relacionadas ao trabalho no contexto brasileiro. Mais especificamente, exploramos os seguintes consequentes: satisfação com a vida, satisfação com o trabalho, engajamento no trabalho e saúde psicológica.

Este estudo soma-se ao realizado por Gazica e Spector (2015), na medida em que se propõe a comparar resultados de indivíduos com chamados ocupacionais atendidos, não atendidos e que não perceberam nenhum chamado ocupacional. A diferença central reside no contexto socioeconômico no qual o estudo é realizado, porque, com base na literatura existente, tal mudança de contexto pode influenciar nos resultados. É pos- 
sível que no cenário brasileiro, em razão de uma maior escassez de oportunidades em relação a países desenvolvidos, não atender a um chamado seja mais comum. Pode-se sugerir também que o alto uso das redes sociais no Brasil (POUSHTER, 2016) permite um contato frequente com as realizações declaradas alheias, o que pode despertar um sentimento de frustração (OLLIER-MALATERRE; ROTHBARD; BERG, 2013).

Sendo assim, este estudo contribui para a construção de um conjunto de evidências mais robustas a respeito das consequências de se possuir um chamado ocupacional atendido, não atendido ou de não ter um chamado ocupacional percebido. Possibilita também que a literatura sobre o chamado seja enriquecida com evidências provenientes de um país em condições contextuais distintas das que estão presentes na literatura até o momento. Como contribuição prática, este estudo pode ajudar na elaboração de estratégias de enfrentamento aos prejuízos evidenciados nos resultados, colaborando para deixar mais nítidos quais são os principais problemas apontados pelos participantes neste estudo.

\section{REFERENCIAL TEÓRICO}

A literatura considera duas abordagens principais para o entendimento sobre chamados ocupacionais: a abordagem existencial e a secular (DUFFY; DIK, 2013). Ambas concordam que um chamado ocupacional é uma ocupação que atrai indivíduos e os faz sentir a vida como dotada de significado, porém discordam sobre a origem das motivações que levam o indivíduo a prosseguir nesse caminho. A abordagem existencial inclina-se para a motivação religiosa ou espiritual para perseguir um determinado chamado. Por outro lado, a abordagem secular, sob uma perspectiva mais ampla, foca na significância e no prazer do indivíduo em fazer parte do chamado que possui, e esse prazer ser oriundo de uma realização pessoal ou de um impulso religioso (GAZICA; SPECTOR, 2015). Nesta pesquisa foi adotada para o desenvolvimento a revisão da literatura, e para a pesquisa empírica a abordagem secular, posto que ela é mais abrangente e atende melhor às diferentes origens de um chamado ocupacional.

A apresentação dos conceitos de chamado ocupacional e significado no trabalho também se mostra necessária. Como já abordado anteriormente, neste trabalho adota-se a definição de chamado ocupacional como uma ocupação na qual um indivíduo se sente motivado a buscar, espera que seja prazerosa, dotada de significado e que seja uma parte central de sua identidade (WRZESNIEWSKI et al., 1997). Por sua vez, para significado no trabalho adota-se a seguinte definição: "o valor de um objetivo ou propósito de trabalho, julgado pelos próprios ideais ou padrões do indivíduo" (MAY; GILSON; HARTER, 2004, p. 14). Assim, viver uma vida com abundância de significado por meio do chamado ocupacional é uma experiência particular e subjetiva de cada indivíduo, podendo acarretar benefícios para os que experimentam essa realidade (GAZICA; SPECTOR, 2015). Os benefícios são gerados à medida que um chamado ocupacional anda junto com a percepção de significado, ou seja, um chamado ocupacional traz benefícios na medida em que o indivíduo vê nessa vocação algum significado para sua vida (DUFFY et al., 2017). Como definição de indivíduo motivado, é usada a proposta por Ryan e Deci (2000), para quem uma pessoa motivada é "alguém que é energizado ou ativado em direção a um fim". 


\section{O Contexto Socioeconômico}

Na literatura sobre o chamado ocupacional, a maior parte dos estudos foram feitos nos Estados Unidos tendo como amostras estudantes universitários e adultos (DOUGLASS; DUFFY; AUTIN, 2016) e em países desenvolvidos como Canadá (DOMENE, 2012) e Alemanha (HIRSCHI; HERRMANN, 2013). Pesquisas anteriores, contudo, mostraram que um chamado pode ser experimentado em vários campos, como contextos socioeconômicos (AUTIN et al., 2017).

Pesquisas feitas em outros países apresentaram resultados os quais podem ser explicados pelo contexto socioeconômico. Por exemplo, Douglas, Duffy e Autin (2016) compararam os resultados obtidos de amostras compostas por estadunidenses e indianos. Segundo os autores, a prevalência da pobreza na Índia pode ter influenciado nos resultados dos trabalhadores indianos, pois os que foram capazes de atingir um emprego podem considerá-lo como uma oportunidade de satisfazer suas necessidades básicas e não como uma fonte de realização pessoal, deixando a busca por um chamado. Os autores ainda encontraram que os indianos que vivem um chamado possuem níveis mais baixos de satisfação no trabalho e na vida em comparação com os estadunidenses que vivem um chamado. Uma possível explicação foi dada com base nas diferenças nacionais de orientação do trabalho porque para os estadunidenses este é visto como um dos principais propósitos de existências dos indivíduos, impactando mais neles.

Outro estudo realizado na China também evidenciou diferenças na conceitualização sobre o chamado e apontou a cultura local como motivo para a diferença em relação aos estadunidenses (ZHANG et al., 2015). Segundo os autores, valores da cultura chinesa afetaram a percepção do chamado, fazendo com que alguns indivíduos percebessem um chamado como um conceito muito elevado para se referir a uma busca "mundana". Desta forma, uma mudança de contexto pode impactar nos resultados encontrados.

No caso deste estudo, foi escolhido o contexto brasileiro, caracterizado como um país emergente economicamente, ainda que em uma crise financeira que se estende há anos e acarreta sérias limitações em termos de possibilidade de trabalho para os cidadãos residentes nesse território (POCHMANN, 2018).

\section{Chamados Ocupacionais Percebidos e Atendidos}

Os estudos sobre o chamado ocupacional indicam que existe uma diferença entre perceber e viver um chamado ocupacional. Perceber um chamado está relacionado ao grau com que um indivíduo acredita ser chamado para uma determinada carreira, ou seja, o grau no qual o chamado está se manifestando em sua vida. Por outro lado, viver um chamado ocupacional reflete o grau em que o indivíduo está atualmente envolvido em um emprego que atende a esse chamado (DUFFY et al., 2017; DUFFY; AUTIN, 2013).

A literatura tem apontado constantemente e de forma robusta os benefícios que aqueles que vivem um chamado ocupacional atendido experimentam. Por exemplo, estudos têm apontado que um chamado ocupacional atendido pode resultar em benefícios profissionais, como maior satisfação no trabalho (STEGER; DIK; DUFFY, 2012; DUFFY et al., 2012; DUFFY et al., 2016), uma preparação superior na carreira, maior autoeficácia (HIRSCHI; HERRMANN, 2013; PRASKOVA; HOOD; CREED, 2014), maior adaptabilidade (DOUGLASS; DUFFY, 2015) e melhor desenvolvimento profissional (DUFFY; 
SEDLACEK, 2007). Além disso, um chamado atendido pode proporcionar uma maior empregabilidade quando está relacionado a um desenvolvimento profissional proativo (LYSOVA, 2018) e tornar a pessoa mais propensa a prosseguir na carreira para a qual se sente chamada (GALLES; LENZ, 2013). Outros benefícios, além dos profissionais, também têm sido apontados pela literatura como efeitos positivos de ter um chamado ocupacional atendido. Estudos mostram que viver um chamado ocupacional está diretamente ligado ao bem-estar (STEGER; DIK; DUFFY, 2012; DUFFY et al., 2017) e à saúde psicológica (GAZICA; SPECTOR, 2015). A literatura tem mostrado que as organizações também se beneficiam com a contratação de funcionários que possuem um chamado ocupacional atendido, uma vez que eles tendem a ter mais compromisso com a sua carreira e a contribuir mais com a organização por meio do seu trabalho (KIM et al., 2018). Como, entretanto, nem todas as pessoas conseguem viver seus chamados ocupacionais, fez-se necessário considerar também o que acontece quando não se consegue atender ao ideal de viver um chamado ocupacional que proporciona um senso de significância.

\section{Chamados Ocupacionais Não Percebidos e Não Atendidos}

Antes de abordar este tópico surge a necessidade de fazer uma distinção entre o que foi considerado como não perceber e como não atender a um chamado ocupacional. Este estudo considerou um chamado ocupacional uma ocupação na qual um indivíduo se sente motivado a buscar, espera que seja prazerosa, dotada de significado e que seja uma parte central de sua identidade (WRZESNIEWSKI et al., 1997), e pode-se afirmar que um indivíduo sem um chamado ocupacional é alguém que não percebe significado nem como parte central de sua identidade. Já um indivíduo com um chamado não atendido é alguém que percebeu um chamado ocupacional, mas não está a vivenciá-lo.

Embora a literatura existente sobre o chamado ocupacional aponte que perceber e viver um chamado resulta em diversos benefícios para o indivíduo, tem também mostrado que ter um chamado e não conseguir vivenciá-lo pode acarretar prejuízos para o trabalho, vida e saúde dos que experimentam essa realidade (GAZICA; SPECTOR, 2015). Por exemplo, ainda segundo os autores, não atender a um chamado ocupacional pode resultar em danos para a saúde psicológica. A literatura também aponta consequências para a vida profissional. Indivíduos que possuem um chamado o qual não estão vivendo em seu atual trabalho, tendem a ter maiores intenções de se demitirem (DUFFY; DIK, 2013) e possuem maior tendência a se arrependerem no longo prazo de suas decisões de carreira (BERG; GRANT; JOHNSON, 2010). Além disso, o caminho para alcançar o chamado ocupacional também pode ser prejudicial. A busca por um chamado ocupacional, especialmente quando malsucedida, pode trazer consequências negativas nas relações interpessoais (CARDADOR; CAZA, 2012), estresse (VAN VUUREN, 2017) e diminuir a satisfação da vida se o indivíduo perceber que não tem a oportunidade de viver o chamado ou se não houver nele um senso de significado de vida (DUFFY et al., 2016). Por fim, Gazica e Spector (2015) mostraram que indivíduos sem um chamado ocupacional possuem menos prejuízos no tocante à qualidade de vida, desempenho no trabalho e saúde do que aqueles que experimentam um chamado ocupacional sem resposta. Por sua vez, os que experimentam um chamado ocupacional atendido possuem mais benefícios quanto à qualidade de vida, desempenho no trabalho e saúde. 


\section{Construção Teórica dos Chamados Ocupacionais}

O chamado ocupacional, embora seja um tema crescente na literatura, ainda não dispõe de uma literatura própria madura (DUFFY; DIK, 2013), e, consequentemente, a maior parte da literatura publicada usa como base outras teorias (GAZICA; SPECTOR, 2015). Duas exceções são os trabalhos de Dobrow (2013) e Hall e Chandler (2005), conquanto não expliquem como um indivíduo pode experimentar um chamado ocupacional sem resposta (GAZICA; SPECTOR, 2015). Para isso, por se assemelhar ao trabalho de Gazica e Spector (2015), este estudo usou a mesma base teórica dos autores, a teoria da autodeterminação, ou self-determination theory, que, a partir de agora, será chamada de SDT, pois esta fornece uma melhor compreensão da motivação. A SDT é considerada uma teoria macro sobre a motivação humana que evoluiu a partir das pesquisas sobre motivações intrínsecas e extrínsecas, e hoje se expandiu para vários domínios da vida, entre eles o trabalho (DECl; OLAFSEN; RYAN, 2017).

A SDT propõe duas formas de motivação: a autônoma e a controlada (GAGNÉ; $\mathrm{DECl}, 2005)$. Ainda segundo os autores, a autônoma pode se dar pelo simples interesse na atividade (motivação intrínseca) e/ou porque o valor e a obrigatoriedade na realização da atividade foram integrados (motivação extrínseca integrada), ou seja, além do prazer na atividade há um fator externo que ajuda na motivação para realizar uma determinada atividade. Por outro lado, a motivação controlada consiste em regulação externa e motivação extrínseca. Desta forma, o grau de motivação está diretamente relacionado ao grau em que alguém se sente coagido por fatores externos. Para este estudo sobre chamado ocupacional, com base nas definições já apresentadas acerca do assunto, será considerada a motivação autônoma, uma vez que o chamado ocupacional é algo subjetivo e particular de cada indivíduo.

A literatura da SDT mostra que indivíduos que possuem motivação autônoma elevada tendem a manifestar níveis mais altos de desempenho e satisfação no trabalho, maior compromisso organizacional e níveis mais baixos de angústia psicológica e Burnout do que aqueles com menor grau de motivação autônoma (GAZICA; SPECTOR, 2015; FERNET; GAGNÉ; AUSTIN, 2010; KUVAAS, 2008; LAM; GURLAND, 2008). Ainda, funcionários que possuem maior motivação autonôma estão menos propensos a sofrer dificuldades psicológicas na presença de uma demanda ameaçadora no emprego (TRÉPANIER; FERNET; AUSTIN, 2013). Destaca-se, também, que funcionários com motivação autônoma realizam seu trabalho porque encontram prazer e satisfação na atividade (GAZICA; SPECTOR, 2015). Assim, com base na abordagem secular, nas definições colocadas e na SDT, tem-se uma base sólida para fundamentar o chamado ocupacional e as diferentes percepções dos indivíduos sobre ele.

Com base na SDT e na literatura apresentada sobre o chamado ocupacional, é possível observar os benefícios de se ter um chamado atendido e os malefícios de não conseguir atender ao chamado. Também mostra que não perceber chamado ocupacional algum pode se mostrar menos prejudicial ao indivíduo que possuir um chamado e não atender a ele. Assim, observando a literatura sob a lente da SDT, formulam-se as seguintes hipóteses: 
Hipótese 1: Aqueles que vivenciam um chamado ocupacional possuem níveis mais altos de engajamento no trabalho, satisfação no trabalho e com a vida e níveis mais baixos, angústia psicológica e intenções de saída, do que aqueles que estão experimentando um chamado ocupacional não atendido.

Hipótese 2: Aqueles que experimentam um chamado ocupacional possuem níveis mais altos de engajamento no trabalho, satisfação no trabalho e com a vida e níveis mais baixos, angústia psicológica e intenções de saída, do que aqueles que consideram não possuir um chamado ocupacional.

Hipótese 3: Aqueles que vivenciam um chamado ocupacional não atendido apresentam menores níveis de engajamento no trabalho, satisfação no trabalho e com a vida e níveis mais baixos, angústia psicológica e intenções de saída, do que aqueles que consideram não possuir um chamado ocupacional.

\section{METODOLOGIA}

\section{Natureza da Pesquisa}

O estudo possui uma abordagem quantitativa, descritiva e com corte transversal. O caráter quantitativo se dá pelas percepções dos indivíduos acerca do chamado ocupacional, e as consequências por eles observadas serão representadas de forma direta por dados numéricos, possibilitando uma análise estatística a partir dos dados coletados. 0 traço descritivo é em razão da finalidade da pesquisa em buscar compreender como a percepção do chamado ocupacional pode afetar tanto de forma positiva quanto negativa um indivíduo. E a característica transversal é porque os dados foram coletados em um mesmo intervalo de tempo em um único ponto da trajetória de vida dos participantes (HAIR JUNIOR et al., 2003).

\section{Amostra}

A amostra da pesquisa foi composta por funcionários públicos brasileiros das esferas municipais, estaduais e federal. Esse público foi escolhido em virtude dos salários acima da média de mercado e da estabilidade que possui nos cargos. Segundo Douglass, Duffy e Autin (2016), a pobreza pode ser um fator determinante na decisão de não buscar um chamado ocupacional, pois leva os indivíduos a priorizarem a satisfação de suas necessidades básicas. Sendo assim, o fato de o Brasil ser um país emergente pode levar os indivíduos a abrirem mão de seus chamados para terem estabilidade e bons salários em ocupações não tão atrativas. Por outro lado, muitos cargos públicos também são fontes de realização pessoal e de significância para muitos indivíduos.

Ao todo, participaram da pesquisa 274 servidores públicos das esferas municipais, estaduais e federal, dos quais 125 eram do sexo masculino e 149 do feminino. A média de idade dos participantes foi de 39 anos, e a renda média foi de $\mathrm{R} \$ 5.757,74$ reais, conforme Tabela 1.

\section{Técnica de Coleta de Dados}

Para coletar os dados foi utilizado um questionário fechado com 49 questões, formado por 7 escalas tipo Likert para medir cada variável e por um conjunto de questões demográficas. Por se tratar de escalas construídas na língua inglesa, o questionário foi 
traduzido pelos autores e enviado para uma nativa estadunidense fluente em português para fazer a tradução reversa, com a finalidade de testar a consistência da tradução feita. Depois desse procedimento, o questionário foi passado para uma plataforma on-line: o "Google Forms". Na sequência, foi realizado um pré-teste com 15 participantes que não identificaram nenhum problema no questionário. Posteriormente à validação do questionário no pré-teste, ele foi enviado por e-mail para a Escola do Serviço Público do Estado do Espírito Santo (Esesp), que o imprimiu e distribuiu para servidores públicos que participavam de seus cursos regulares de capacitação. A coleta ocorreu no segundo semestre de 2018.

\section{Escalas}

As escalas escolhidas já foram utilizadas e validadas anteriormente, por exemplo no estudo de Gazica e Spector (2015), e visavam a medir todas as variáveis envolvidas nas hipóteses deste estudo. Cada uma das escalas utilizada está descrita a seguir.

A escala tipo Likert, usada para medir quanto aos respondentes que percebem um chamado ocupacional, mas não vivem, foi a escala desenvolvida por Gazica (2014). Essa escala possui seis opções de respostas, variando de 1 (discordo fortemente) a 6 (concordo fortemente). Para avaliar o grau em que cada respondente percebe seu emprego atual como um chamado ocupacional, foi utilizada uma versão adaptada da escala tipo Likert usada por Dobrow e Tosti-Kharas (2011). Tal adaptação também foi empregada por Gazica e Spector (2015). A escala possui 12 itens e tem 6 opções de respostas, variando de 1 (discordo fortemente) a 6 (concordo fortemente).

A escala tipo Likert, usada para medir a satisfação com a vida, foi desenvolvida por Diener et al. (1985). A escala possui cinco itens e teve seis opções de respostas, variando de 1 (discordo fortemente) a 6 (concordo fortemente). Para avaliar a satisfação no trabalho foi utilizada a subescala de satisfação no trabalho do Questionário de Avaliação Organizacional de Michigan (CAMMANN, 1983). Essa escala tipo Likert possui três itens e teve seis opções de respostas, variando de 1 (discordo fortemente) a 6 (concordo fortemente). Para medir o engajamento no trabalho dos participantes, foi usada a escala tipo Likert desenvolvida por Schaufeli, Bakker e Salanova (2006). Essa escala possui seis opções de respostas, variando de 1 (nunca) a 6 (sempre).

Para avaliar os sintomas psicológicos relacionados ao trabalho, foi empregada a escala Likert desenvolvida por Caplan et al. (1975), a qual consiste num total de 12 itens. Os respondentes foram instruídos a responder com qual frequência eles sofrem os sintomas psicológicos. Essa escala possui seis opções de respostas, variando de 1 (não) a 6 (várias vezes por dia).

Para avaliar a intenção de se demitir foi utilizado um único item. Os participantes responderam com qual frequência eles pensam em deixar seu trabalho (SPECTOR; DWYER; JEX, 1988). As opções de respostas variaram de 1 (nunca) a 6 (extremamente frequente). Por fim, cada participante respondeu a perguntas demográficas sobre sua idade, estado civil, sexo, tempo no emprego, renda e escolaridade. 


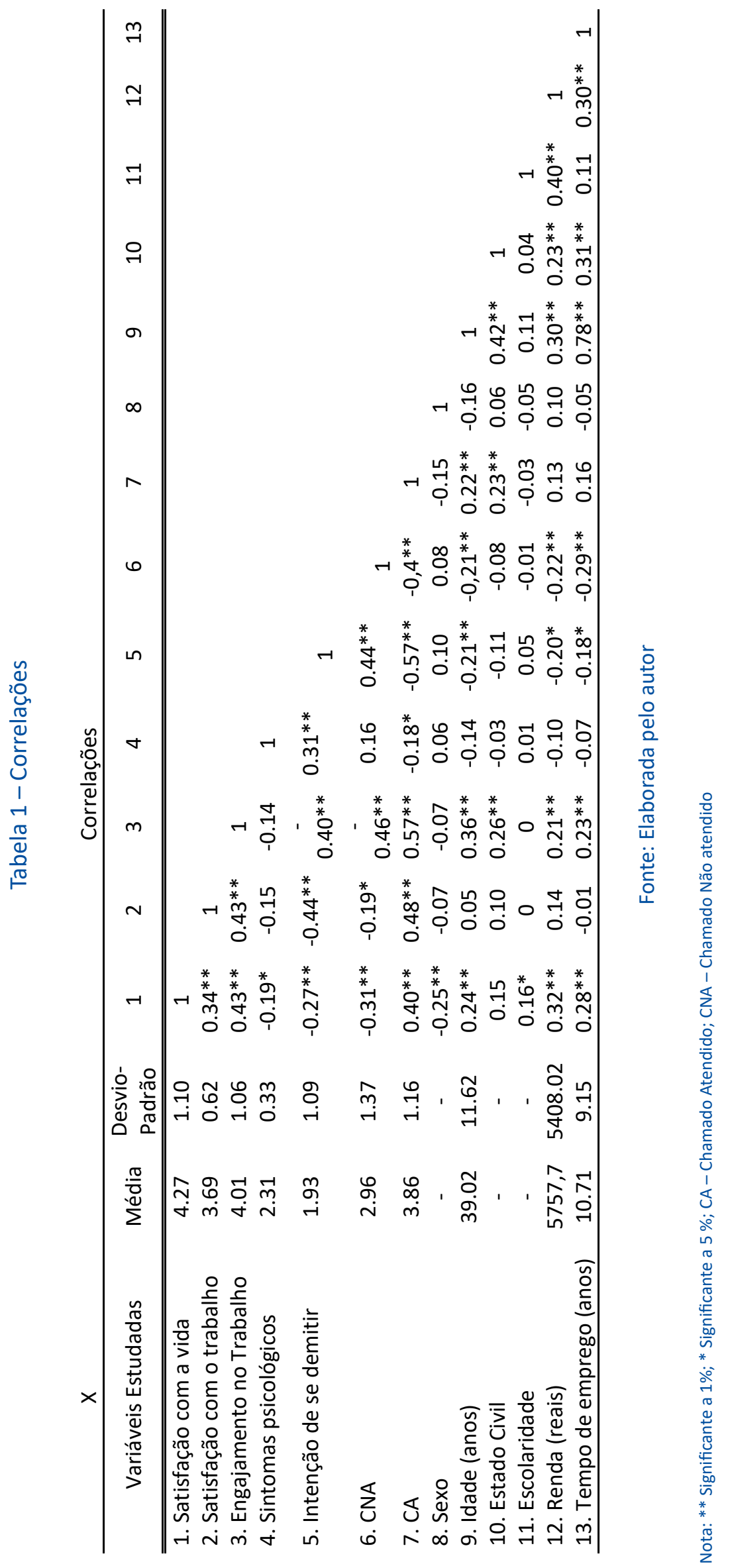




\section{RESULTADOS}

A Tabela 1 apresenta as correlações entre as variáveis focais deste estudo, bem como a média e o desvio-padrão. Para testar as três hipóteses deste estudo foi necessário dividir as amostras em quatro grupos para fazer as comparações que se propõem. Cada participante foi categorizado de acordo com o resultado que ele ou ela obteve na escala do grupo de chamadas ocupacionais não atendidas e escala de chamadas ocupacionais atendidas. Foi estabelecida uma média de acordo com o número atribuído às respostas nas escalas e, desta forma, à menor resposta de concordância na escala (Concordo um pouco) foi atribuído o número quatro.

Sendo assim, os participantes que obtiveram média maior que quatro somente na escala do chamado atendido, foram colocados no grupo "Chamado atendido", o qual será mencionado, a partir de agora, como $C A(N=73)$. De igual modo, os participantes com média maior que quatro somente na escala do chamado não atendido, foram colocados no grupo "Chamado não atendido", o qual será mencionado a partir de agora como CNA ( $\mathrm{N}=41)$. Os participantes que obtiveram média inferior a quatro em ambas as escalas foram colocados no grupo do "Sem chamado", o qual será mencionado a partir de agora como SC ( $N=39$ ). Por fim, aqueles participantes com média superior a quatro em ambas as escalas foram colocados no "Grupo duplo" ( $N=121)$, que captura participantes, os quais experimentam uma chamada com resposta e uma chamada sem resposta. O grupo duplo foi excluído de mais interpretações e análises.

Uma vez definidos os grupos, foram realizados testes unidirecionais de análise de variância (Anova) usando o software R-Project for statistical computing, com o objetivo de avaliar as diferenças entre os três grupos de interesse deste estudo, indivíduos com chamado ocupacional atendido, com chamado ocupacional não atendido e sem chamado ocupacional percebido. Conforme mostrado na Tabela 2, os resultados indicaram diferenças significativas de grupo em todas as variáveis focais deste estudo. Em seguida, foi realizada uma série de testes Duncan para comparar os três grupos em cada variável independente.

A hipótese 1 foi totalmente confirmada, pois os indivíduos com um chamado ocupacional atendido apresentam maiores níveis de satisfação no trabalho (CA: $M=3,95$; $D P=0,47$; $C N A: M=3,53$; $D P=0,69 ; p<0.00$ ), engajamento no trabalho ( $C A: M=4,59$; $D P=0,88 ; C N A: M=3,40 ; D P=0,87 ; p<0.00$ ), maior satisfação com a vida ( $C A: M=4,73$; $D P=0,79$; $C N A: M=3,80 ; D P=1,17 ; p<0.00)$, menores níveis de sintomas psicológicos relacionados ao trabalho ( $C A: M=2,24 ; D P=0,26 ; C N A: M=2,43 ; D P=0,41 ; p<0.01$ ), e menor intenção de se demitir que indivíduos que experimentam um chamado ocupacional não atendido ( $C A: M=1,41 ; D P=0,83 ; C N A: M=2,61 ; D P=1,14 ; p<0.00$ ).

Por sua vez, a hipótese 2 foi totalmente confirmada, exceto pelos sintomas psicológicos. Os resultados mostraram que indivíduos com um chamado ocupacional atendido apresentam maiores níveis de satisfação no trabalho ( $C A: M=3,95 ; D P=0,47$; $S C: M=3,38 ; D P=0,61 ; p<0.00$ ), engajamento no trabalho ( $C A: M=4,59 ; D P=0,88$; $S C$ : $M=3,56 ; D P=1,0 ; p<0.00$ ), maior satisfação com a vida (CA: $M=4,73 ; D P=0,79 ; S C$ : $M=3,92 ; D P=1,20 ; p<0.00)$, e menor intenção de se demitir que aqueles que não per- 
cebem um chamadoocupacional (CA: $M=1,41 ; D P=0,83 ; S C: M=2,18 ; D P=1,02 ; p<0.00$ ). Não houve diferença significativa, contudo, em relação aos sintomas psicológicos relacionados ao trabalho ( $C A: M=2,24 ; D P=0,26 ; S C: M=2,33 ; D P=0,34 ; p>0.1$ ).

Por fim, a hipótese 3 foi totalmente rejeitada, exceto pela intenção de se demitir, que foi confirmada. Os resultados não apontaram diferenças significativas entre indivíduos com chamado ocupacional não atendido e aqueles que não perceberam um chamado em relação à satisfação no trabalho (CNA: $M=3,53 ; D P=0,69 ; S C: M=3,38 ; D P=0,61$; $p>0.1$ ), engajamento no trabalho (CNA: $M=3,40 ; D P=0,87 ; S C: M=3,56 ; D P=1,0 ; p>0.1$ ), satisfação com a vida (CNA: $M=3,80 ; D P=1,17$; $S C$ : $M=3,92 ; D P=1,20 ; p>0.1$ ) e sintomas psicológicos relacionados ao trabalho (CNA: $M=2,43$; $D P=0,41 ; S C: M=2,33$; $D P=0,34$; $p>0.1$ ). A única diferença significativa foi em relação à intenção de se demitir; aqueles que experimentam um chamado não atendido apresentaram maiores intenções de se demitirem que aqueles que não possuem um chamado atendido, confirmando parte da hipótese 3 (CNA: $M=2,61 ; D P=1,14 ; S C: M=2,18 ; D P=1,02 ; p<0.00)$.

Tabela 2 - Anova e teste Duncan

\begin{tabular}{|c|c|c|c|c|}
\hline \multicolumn{5}{|c|}{$\mathrm{X}$ Anova e teste Duncan } \\
\hline \multirow[b]{2}{*}{ Variáveis de interesse } & & & \multicolumn{2}{|l|}{ ANOVA } \\
\hline & F-ratio & valor $p$ & $\begin{array}{l}\text { Média dos gru- } \\
\text { pos }\end{array}$ & $\begin{array}{l}\text { Desvio Padrão dos } \\
\text { grupos }\end{array}$ \\
\hline Engajamento no trabalho & \multirow{4}{*}{29.115 .728} & \multirow{4}{*}{0.00} & & \\
\hline CA & & & $4.59 a$ & 0.88 \\
\hline CNA & & & $3.40 \mathrm{~b}$ & 0.87 \\
\hline SC & & & $3.56 b$ & 1.00 \\
\hline Intenção de se demitir & \multirow{4}{*}{21.802 .417} & \multirow{4}{*}{0.00} & & \\
\hline CA & & & $1.41 \mathrm{c}$ & 0.83 \\
\hline CNA & & & $2.61 a$ & 1.14 \\
\hline SC & & & $2.18 b$ & 1.02 \\
\hline Sintomas psicológicos & \multirow{4}{*}{4.427 .649} & \multirow{4}{*}{0.01} & & \\
\hline CA & & & $2.24 b$ & 0.26 \\
\hline CNA & & & $2.43 a$ & 0.41 \\
\hline SC & & & $2.33 a b$ & 0.34 \\
\hline Satisfação com trabalho & \multirow{4}{*}{15.106 .782} & \multirow{4}{*}{0.00} & & \\
\hline $\mathrm{CA}$ & & & $3.95 a$ & 0.47 \\
\hline CNA & & & $3.53 b$ & 0.69 \\
\hline SC & & & $3.38 b$ & 0.61 \\
\hline Satisfação com a vida & \multirow{4}{*}{ 14.440.761 } & \multirow{4}{*}{0.00} & & \\
\hline CA & & & $4.73 a$ & 0.79 \\
\hline CNA & & & $3.80 \mathrm{~b}$ & 1.17 \\
\hline SC & & & $3.92 b$ & 1.20 \\
\hline
\end{tabular}

Nota: CA - Chamado Atendido; CNA - Chamado não atendido; SC - Sem chamado.

Fonte: Elaborada pelo autor. 


\section{DISCUSSÃO}

Este estudo se propôs a comparar três grupos de indivíduos com diferentes percepções acerca do chamado ocupacional, um grupo com indivíduos com chamado atendido, um com indivíduos com chamado não atendido e, por fim, um com indivíduos que não possuem um chamado ocupacional em termos de satisfação com a vida, satisfação com o trabalho, engajamento no trabalho, sintomas psicológicos relacionados à carreira e intenção de se demitir. Com essa comparação buscou-se sustentar a ideia de que possuir um chamado ocupacional pode ser uma faca de dois gumes, caso se confirmasse o achado de que pessoas com chamados não atendidos apresentassem resultados mais insatisfatórios que os outros dois grupos.

Os resultados encontrados confirmaram totalmente a hipótese 1 . Os achados mostram que os indivíduos que vivenciam um chamado atendido tendem a reportar níveis mais elevados de resultados positivos para a vida, o trabalho e para a saúde psicológica do que aqueles que possuem um chamado não atendido. Tais achados vão ao encontro da literatura existente e aos achados de Gazica e Spector (2015). Os resultados são consistentes com a teoria da autodeterminação (SDT). Segundo a SDT, aqueles que contemplam suas competências, autonomia e necessidades de relacionamento, experimentam benefícios psicológicos, funcionamento ótimo e bem-estar (DECl; OLAFSEN; RYAN, 2017). Por sua vez, o chamado ocupacional fornece aos indivíduos significado na vida, e os benefícios são gerados à medida que um chamado ocupacional anda junto com a percepção de significado (DUFFY et al., 2017). Desta forma, os resultados encontrados na hipótese 1 são consistentes com a SDT e com a literatura sobre o chamado, uma vez que os indivíduos com um chamado ocupacional atendido mostram níveis mais elevados de engajamento no trabalho, satisfação com o trabalho e com a vida que os que experimentam um chamado não atendido e os que não possuem um chamado.

A hipótese 2 foi parcialmente confirmada pelos resultados encontrados, exceto pelos sintomas psicológicos. Os achados mostram que os indivíduos que vivenciam um chamado atendido tendem a reportar níveis mais elevados de resultados positivos para vida e trabalho do que aqueles que não percebem um chamado. A exceção encontrada foram os sintomas psicológicos, nos quais não houve diferença significativa entre os dois grupos. Os resultados encontrados são, em grande parte, consistentes com a literatura sobre o chamado ocupacional e com a SDT (GAGNÉ; DECl, 2005). Como na hipótese 1 , os indivíduos que possuem um chamado atendido recebem os benefícios gerados por causa dessa realização. Desta forma, os achados estão de acordo com a SDT, exceto pelos benefícios psicológicos. Uma possível explicação pode ser o contexto de crise financeira atual no Brasil. Nesse cenário de escassez de oportunidades, é possível que o indivíduo que não percebeu um chamado não se sinta frustrado com seu atual emprego, pois sua realidade ainda é melhor que a de muitos a sua volta (FELIX; CAVAZOTTE, 2019). A estabilidade que os funcionários públicos possuem, somada a um cenário de falta de emprego e crise financeira, pode fazer com que o indivíduo se veja numa situação privilegiada, reduzindo, assim, os resultados psicológicos negativos.

Por outro lado, na hipótese 3, diferentemente dos achados de Gazica e Spector (2015), os resultados encontrados não mostraram diferenças significativas entre aqueles que vivem um chamado não atendido e aqueles que não perceberam um chamado, 
com exceção da intenção de se demitir, quando os indivíduos com um chamado não atendido mostraram maiores intenções de se demitir. Uma possível explicação pode se dar pela prevalência da pobreza no Brasil, intensificada por um sentimento de escassez de oportunidades trazido pela crise financeira, fazendo com que o indivíduo veja o trabalho como um privilégio e como um meio para satisfazer suas necessidades básicas, e não como fonte de realização e significado (FELIX; CAVAZOTTE, 2019). Tal contexto pode ser intensificado no serviço público, pois este oferece estabilidade, sendo, assim, alvo de muitos indivíduos que buscam fugir das incertezas, e é possível que esses indivíduos tenham prescindido de seus chamados pela segurança e estabilidade. Desta forma, embora possua um chamado não atendido, o indivíduo pode se sentir em uma posição comparativamente não tão desfavorecida na sociedade ao ver muitas pessoas em condições não ideais de carreira (DUFFY et al., 2016). Assim, ao ver menos pessoas atendendo a chamados, é possível que o indivíduo reduza os efeitos negativos de não atender a seu chamado, pois tais efeitos podem possuir natureza comparativa, a ponto de experimentar o sucesso de forma similar a quem não tem um chamado. A SDT também pode fornecer uma possível explicação para rejeição parcial da hipótese 3 . Uma vez que no Brasil o serviço público é visto como fonte de autonomia e status, é possível que tal percepção tenha amenizado os efeitos negativos de se experimentar um chamado ocupacional não atendido.

\section{CONCLUSÃO}

Este é o primeiro estudo a comparar os grupos chamado atendido, chamado não atendido e sem chamado no contexto brasileiro. Os resultados encontrados sugerem que os indivíduos com chamado ocupacional atendido apresentam maiores benefícios do que indivíduos com chamados não atendidos e sem chamados em relação ao trabalho, à vida e à saúde psicológica. De forma inesperada, os resultados não apontaram diferenças significativas entre os que possuem um chamado não atendido e os que não possuem um chamado, exceto pela intenção de se demitir, contrariando os resultados encontrados em países desenvolvidos e rejeitando parcialmente a hipótese 3. Este estudo contribui para os estudos acerca do chamado ao explorar um novo contexto socioeconômico e trazendo resultados que divergem dos encontrados em estudos feitos em outros contextos. Esta pesquisa reforça a literatura no sentido de apontar que ter um chamado somente é benéfico à medida que o indivíduo consegue vivenciá-lo. Os resultados encontrados são consistentes com a SDT, na medida em que um indivíduo com um chamado atendido possui melhores níveis em relação ao engajamento no trabalho, à satisfação com o trabalho e à vida, menor intenção de se demitir e níveis baixos de sintomas psicológicos que aqueles que possuem um chamado não atendido.

Este trabalho foi realizado em um momento no qual o Brasil atravessava uma crise financeira que, como consequência, gerou uma escassez de oportunidades, e tal contexto pode ter afetado os resultados. Em segundo lugar, a amostra deste estudo consistiu em funcionários públicos, que, em média, possuem salários maiores que os funcionários da iniciativa privada, e possuem, também, estabilidade em seus empregos. Esses fatores podem ter feito com que algumas outras características da população não tenham sido exploradas. Sendo assim, como sugestão para estudos futuros, deve-se realizar tal pesquisa com amostras mais diversas com maior variabilidade em termos de 
recursos econômicos. Por fim, explorar melhor a percepção do brasileiro acerca do chamado ocupacional e aprofundar no impacto que a cultura e o contexto socioeconômico possuem em tal percepção.

Em termos práticos, esta pesquisa apresenta resultados que influenciam tanto a ação de orientadores de carreira quanto de trabalhadores em si. Para orientadores de carreira, o artigo permite uma reflexão a respeito dos possíveis efeitos nefastos da moda de se incentivar que todos os indivíduos nutram a ideia de que precisam ter uma missão de vida profissional e um chamado ocupacional. Embora essa prática possa levar a resultados muito positivos, caso o indivíduo não consiga viabilizar uma carreira nesse sentido, as evidências mostraram um cenário bastante preocupante. Para trabalhadores, sugere-se que adotem um olhar mais crítico e realista quando se virem diante de eventos que os estimulem a desenvolver um chamado ocupacional. Caso, por circunstâncias da vida, a vivência de tal chamado se mostre improvável, recomenda-se não nutrir tais expectativas.

\section{REFERÊNCIAS}

ARNOLD, K. A.; TURNER, N.; BARLING, J.; KELLOWAY, E. K.; MCKEE, M. C. Transformational leadership and psychological well-being: the mediating role of meaningful work. Journal of Occupational Health Psychology, v. 12, n. 3, p. 193, 2007.

AUTIN, K. L.; ALLAN, B. A.; PALANIAPPAN, M.; DUFFY, R. D. Career calling in India and the United States: A cross-cultural measurement study. Journal of Career Assessment, v. 25, n. 4, p. 688-702, 2017.

BERG, J. M.; GRANT, A. M.; JOHNSON, V. When callings are calling: Crafting work and leisure in pursuit of unanswered occupational callings. Organization Science, v. 21, n. 5, p. 973-994, 2010.

CAMMANN, C. Assessing the attitudes and perceptions of organizational members. Assessing organizational change: A guide to methods, measures, and practices, 1983. p. 71-138.

CAPLAN, R. D.; COBB, S.; FRENCH, J. R. Job demands and worker health; main effects and occupational differences. In: Hew Publication (NIOSH), DHEW, 1975.

CARDADOR, M. T.; CAZA, B. B. Relational and identity perspectives on healthy versus unhealthy pursuit of callings. Journal of Career Assessment, v. 20, n. 3, p. 338-353, 2012.

DECI, E. L.; OLAFSEN, A. H.; RYAN, R. M. Self-determination theory in work organizations: The state of a science. Annual Review of Organizational Psychology and Organizational Behavior, v. 4, p. 19-43, 2017.

DIENER, E. D.; EMMONS, R. A.; LARSEN, R. J.; GRIFFIN, S. The satisfaction with life scale. Journal of Personality Assessment, v. 49, n. 1, p. 71-75, 1985.

DOBROW, S. R.; TOSTI-KHARAS, J. Calling: The development of a scale measure. Personnel Psychology, v. 64, n. 4, p. 1.001-1.049, 2011.

DOBROW, S. R. Dynamics of calling: A longitudinal study of musicians. Journal of Organizational Behavior, v. 34, n. 4, p. 431-452, 2013.

DOMENE, J. F. Calling and career outcome expectations: The mediating role of self-efficacy. Journal of Career Assessment, v. 20, n. 3, p. 281-292, 2012.

DOUGLASS, R. P.; DUFFY, R. D. Calling and career adaptability among undergraduate students. Journal of Vocational Behavior, v. 86, p. 58-65, 2015.

DOUGLASS, R. P.; DUFFY, R. D.; AUTIN, K. L. Living a calling, nationality, and life satisfaction: A moderated, multiple mediator model. Journal of Career Assessment, v. 24, n. 2, p. 253-269, 2016.

DUFFY, R. D.; SEDLACEK, W. E. The presence of and search for a calling: Connections to career development. Journal of Vocational Behavior, v. 70, n. 3, p. 590-601, 2007.

DUFFY, R. D.; BOTT, E. M.; ALLAN, B. A.; TORREY, C. L.; DIK, B. J. Perceiving a calling, living a calling, and job satisfaction: Testing a moderated, multiple mediator model. Journal of Counseling Psychology, v. 59, n. 1, p. 50, 2012.

DUFFY, R. D.; AUTIN, K. L. Disentangling the link between perceiving a calling and living a calling. Journal of Counseling Psychology, v. 60, n. 2, p. 219, 2013.

DUFFY, R. D.; DIK, B. J. Research on calling: What have we learned and where are we going? Journal of Vocational Behavior, v. 83, n. 3, p. 428-436, 2013. 
DUFFY, R. D.; DOUGLASS, R. P.; AUTIN, K. L.; ENGLAND, J.; DIK, B. J. Does the dark side of a calling exist? Examining potential negative effects. The Journal of Positive Psychology, v. 11, n. 6, p. 634-646, 2016.

DUFFY, R. D.; ENGLAND, J.; DOUGLASS, R. P.; AUTIN, K. L.; ALLAN, B. A. Perceiving a calling and well-being: Motivation and access to opportunity as moderators. Journal of Vocational Behavior, v. 98, p. 127-137, 2017.

FELIX, B.; CAVAZOTTE, F. When a Calling Goes Unanswered: Exploring the Role of Workplace Personalizations as Calling Enactments. Frontiers in Psychology, v. 10, n. 1.940, p. 1-14, 2019.

FERNET, C.; GAGNÉ, M.; AUSTIN, S. When does quality of relationships with coworkers predict burnout over time? The moderating role of work motivation. Journal of Organizational Behavior, v. 31, n. 8, p. 1.163-1.180, 2010.

GAGNÉ, M.; DECI, E. L. Self-determination theory and work motivation. Journal of Organizational behavior, v. 26, n. 4, p. 331-362, 2005.

GALLES, J. A.; LENZ, J. G. Relationships among career thoughts, vocational identity, and calling: Implications for practice. The Career Development Quarterly, v. 61, n. 3, p. 240-248, 2013.

GAZICA, M. W. Unanswered occupational calling: The development and validation of a new measure. Flórida: University of South Florida, 2014.

GAZICA, M. W.; SPECTOR, P. E. A comparison of individuals with unanswered callings to those with no calling at all. Journal of Vocational Behavior, v. 91, p. 1-10, 2015.

HAIR JUNIOR, J. F.; WOLFINBARGER, M.; MONEY, A. H.; SAMOUEL, P.; PAGE, M. J. Essentials of business research methods. New York, NY: Wiley, 2003.

HALL, D. T.; CHANDLER, D. E. Psychological success: When the career is a calling. Journal of Organizational Behavior, v. 26, n. 2, p. 155-176, 2005.

HIRSCHI, A.; HERRMANN, A. Calling and career preparation: Investigating developmental patterns and temporal precedence. Journal of Vocational Behavior, v. 83, n. 1, p. 51-60, 2013.

KIM, S. S.; SHIN, D.; VOUGH, H. C.; HEWLIN, P. F.; VANDENBERGHE, C. How do callings relate to job performance? The role of organizational commitment and ideological contract fulfillment. Human Relations, $\mathrm{p}$. $0018726717743310,2018$.

KUVAAS, B. A test of hypotheses derived from self-determination theory among public sector employees. Employee relations, v. 31, n. 1, p. 39-56, 2008.

LAM, C. F.; GURLAND, S. T. Self-determined work motivation predicts job outcomes, but what predicts self-determined work motivation? Journal of research in personality, v. 42, n. 4, p. 1.109-1.115, 2008.

LYSOVA, E. I.; JANSEN, P. G.; KHAPOVA, S. N.; PLOMP, J.; TIMS, M. Examining calling as a double-edged sword for employability. Journal of Vocational Behavior, v. 104, p. 261-272, 2018.

MAY, D. R.; GILSON, R. L.; HARTER, L. M. The psychological conditions of meaningfulness, safety and availability and the engagement of the human spirit at work. Journal of Occupational and Organizational Psychology, v. 77, n. 1, p. 11-37, 2004.

OLLIER-MALATERRE, A.; ROTHBARD, N. P.; BERG, J. M. When worlds collide in cyberspace: How boundary work in online social networks impacts professional relationships. Academy of Management Review, v. 38, n. 4, p. 645-669, 2013.

POCHMANN, M. Desempenho econômico conjuntural e a situação recente do trabalho no Brasil. Revista Necat - Revista do Núcleo de Estudos de Economia Catarinense, v. 7, n. 13, p. 12-28, 2018.

POUSHTER, J. Smartphone ownership and internet usage continues to climb in emerging economies. Pew Research Center, v. 22, p. 1-44, 2016.

PRASKOVA, A.; HOOD, M.; CREED, P. A. Testing a calling model of psychological career success in Australian young adults: A longitudinal study. Journal of Vocational Behavior, v. 85, n. 1, p. 125-135, 2014.

ROUSSEAU, D. M.; HO, V. T.; GREENBERG, J. I-deals: Idiosyncratic terms in employment relationships. Academy of Management Review, v. 31, n. 4, p. 977-994, 2006.

RYAN, R. M.; DECI, E. L. Intrinsic and extrinsic motivations: Classic definitions and new directions. Contemporary Educational Psychology, v. 25, n. 1, p. 54-67, 2000.

SCHAUFELI, W. B.; BAKKER, A. B.; SALANOVA, M. The measurement of work engagement with a short questionnaire: A cross-national study. Educational and Psychological Measurement, v. 66, n. 4, p. 701716, 2006.

SPECTOR, P. E.; DWYER, D. J.; JEX, S. M. Relation of job stressors to affective, health, and performance outcomes: a comparison of multiple data sources. Journal of Applied Psychology, v. 73, n. 1, p. 11, 1988.

STEGER, M. F.; DIK, B. J.; DUFFY, R. D. Measuring meaningful work: The work and meaning inventory (WAMI). Journal of Career Assessment, v. 20, n. 3, p. 322-337, 2012. 
TRÉPANIER, S.-G.; FERNET, C.; AUSTIN, S. The moderating role of autonomous motivation in the job demands-strain relation: A two sample study. Motivation and Emotion, v. 37, n. 1, p. 93-105, 2013.

VAN VUUREN, M. Called To Do Meaningful Work: A Blessing or a Curse? Christian Higher Education, v. 16, n. 1-2, p. 47-54, 2017.

WRZESNIEWSKI, A.; MCCAULEY, C.; ROZIN, P.; SCHWARTZ, B. Jobs, careers, and callings: People's relations to their work. Journal of research in personality, v. 31, n. 1, p. 21-33, 1997.

WRZESNIEWSKI, A. Finding positive meaning in work. Positive organizational scholarship: foundations of a new discipline, 2003. p. 296-308.

ZHANG, C.; DIK, B. J.; WEI, J.; ZHANG, J. Work as a calling in China: A qualitative study of Chinese college students. Journal of Career Assessment, v. 23, n. 2, p. 236-249, 2015. 